\section{Le syndrome de grêle court chez l'adulte}

\section{De I'insuffisance intestinale à l'adaptation intestinale}
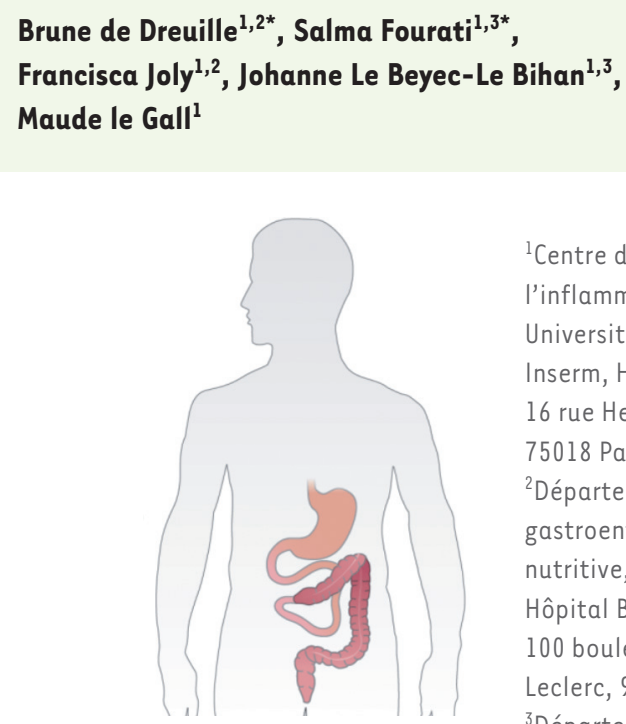

${ }^{1}$ Centre de recherche sur I'inflammation, UMRS 1149, Université de Paris, Inserm, Hôpital Bichat 16 rue Henri Huchard, 75018 Paris, France. ${ }^{2}$ Département de gastroentérologie et assistance nutritive, Hôpital Beaujon, 100 boulevard du général Leclerc, 92110 Clichy, France. ${ }^{3}$ Département de biochimie endocrinienne et oncologique, Hôpital de la Pitié-Salpêtrière,

La longueur de l'intestin grêle est estimée à $600 \mathrm{~cm}$ et celle du côlon à environ 150-200 cm. Le syndrome de grêle court résulte d'une résection de l'intestin grêle, allant de 50 47-83 boulevard de l'hôpital, 75013 Paris, France.

*co-premières auteures maude.le-gall@inserm.fr

à $80 \%$ de sa longueur. La résection peut inclure une partie ou la totalité du côlon. Une longueur résiduelle intestinale en dessous de 150-200 cm, longueur minimale pour assurer les fonctions d'absorption de nutriments, d'eau et d'électrolytes, entraîne une insuffisance intestinale. Celle-ci peut être transitoire ou définitive. Dans ce dernier cas, un soutien nutritionnel devra être instauré, notamment par une nutrition parentérale (par perfusion intraveineuse), le traitement de référence pour répondre aux besoins énergétiques, hydro-électrolytiques et en micronutriments (vitamines, éléments traces) des patients [1, 2].

Une classification fonctionnelle de l'insuffisance intestinale a été établie [2]. Quand elle est chronique, la sévérité peut être évaluée selon le niveau de dépendance à la nutrition parentérale en utilisant une classification en huit catégories, qui tient compte du volume perfusé ainsi que du niveau d'apport énergétique [2]. Si ces classifications permettent d'évaluer le niveau de dépendance au support parentéral, en revanche le degré de sévérité et le pronostic du syndrome de grêle court (SGC) dépendent également de sa cause, de la longueur de grêle restant, et du type de montage anatomique, avec notamment la présence ou non du côlon 


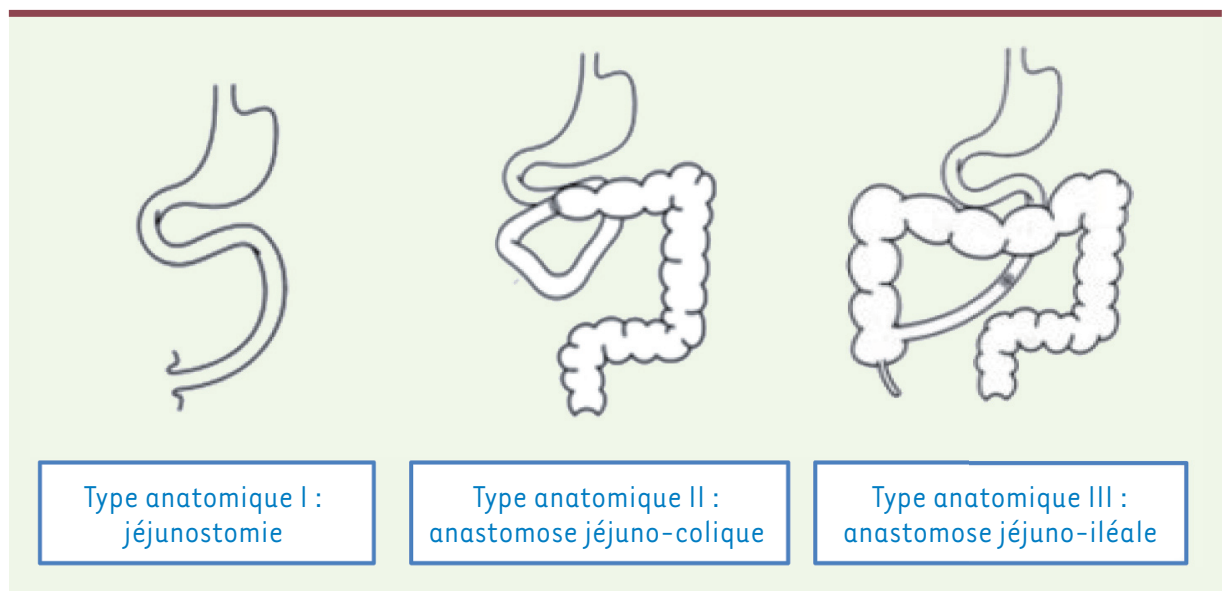

Figure 1. Les trois sous-types anatomiques du syndrome de grêle court. Le type I correspond à une résection d'une grande partie de l'intestin, de l'intestin grêle jusqu'au côlon, avec jéjunostomie. Le type II correspond à une résection de l'intestin grêle avec ablation de l'iléon, de la valve iléo-cæcale et d'une partie du côlon, résultant en une anastomose jéjunocolique. Le type III correspond à une résection de l'intestin grêle dans laquelle une partie de l'iléon est préservée et la valvule iléo-cæcale et le côlon restent intacts, ce qui entraîne une anastomose jéjuno-iléale.

en continuité du grêle restant. Trois types anatomiques de grêle court sont définis (Figure 1). Dans l'entérostomie terminale (type anatomique I), le jéjunum est relié à la paroi abdominale après résection de l'iléon, de la valvule iléo-cæcale et du côlon. Le type anatomique II, qui est majoritaire en France, résulte d'une anastomose jéjuno-colique. L'anastomose jéjuno-iléale définit le type anatomique III. Dans ce cas, la valvule iléo-cæcale et l'ensemble du côlon sont conservés, contrairement au type II.

Les causes les plus fréquentes à l'origine du SGC chez l'adulte peuvent être un infarctus mésentérique (35,8 \% des cas), une maladie de Crohn ${ }^{1}$ (dans $29 \%$ des cas, première cause aux États-Unis), une entérite radique (après une irradiation) ( $9,7 \%$ des cas), des complications chirurgicales $(7,8 \%)$ ou une polypose adénomateuse familiale $^{2}$ ( $4,1 \%$ des cas) [2]. Des tumeurs malignes abdominales ou pelviennes ou une radiothérapie délétère peuvent également conduire à un SGC [3].

La prévalence et l'incidence du syndrome de grêle court ne sont pas connues. II n'existe en effet pas de base de données recensant les patients. Cependant, considérant le nombre de patients sous nutrition parentérale au long cours, des estimations ont pu être réalisées. $\varepsilon$ n Europe, la prévalence de la dépendance à la nutrition parentérale est de 4 cas pour 1 million d'individus [4]. Si on considère que le SGC touche $35 \%$ des patients sous nutrition parentérale à long terme, sa prévalence peut alors être estimée à 1,4 cas pour 1 million de personnes. Cette prévalence a tendance à augmenter et il existe une forte variabilité entre les pays [4]. La proportion de patients qui ont subi une résection intestinale et qui souffrent d'un SGC, est estimée à

\footnotetext{
1 La maladie de Crohn fait partie des maladies inflammatoires chroniques de l'intestin ou MICI.

${ }^{2}$ La polypose adénomateuse familiale est une maladie héréditaire caractérisée par l'apparition de nombreux polypes dans le côlon.
}

$15 \%$. Pour environ $75 \%$ d'entre eux, il s'agit d'une résection de l'intestin grêle importante, mais unique. Les $25 \%$ restants ont été opérés plusieurs fois [5]. L'incidence du SGC serait donc en Europe de deux cas par million d'habitants et par an [2]. Estimée par Orphanet (le serveur d'informations dédié aux maladies rares), la prévalence du SGC en Europe, était, au $1^{\text {er }}$ janvier 2020 , de 20 cas par million d'habitants. La disparité de ces estimations montre la nécessité de l'établissement d'une base de données des patients souffrant d'un SGC afin de définir précisément la prévalence et l'incidence de cette affection.

\section{Les conséquences physiopathologiques d'une résection étendue du grêle : les phases d'adaptation}

À la suite de la ou des résections intestinales menant au SGC, on observe différentes phases de réponse chez les patients, avec des conséquences physiopathologiques qui peuvent s'avérer bénéfiques mais aussi délétères. À côté des processus physiologiques qui surviennent naturellement après la résection d'une partie de l'intestin, des conséquences secondaires, liées à la prise en charge du patient, peuvent également apparaître (Figure 2).

\section{La phase aiguë}

La phase aiguë définit les six semaines qui suivent la résection intestinale étendue. Elle est caractérisée par des pertes hydro-électrolytiques majeures qui sont responsables de diarrhée, de déshydratation et de malabsorption.

\section{Diarrhée et déshydratation}

La perte liquidienne après résection est très importante. Le maintien d'un équilibre hémodynamique est donc un enjeu majeur pour prévenir l'hypovolémie (diminution du volume sanguin) et l'atteinte d'autres organes par hypoperfusion. La physiopathologie de la diarrhée dans le SGC est complexe et plusieurs mécanismes y concourent. La vidange gastrique est généralement plus rapide chez les patients. L'absence d'iléon entraîne en effet la disparition du frein iléal avec, pour conséquence, la perte du 


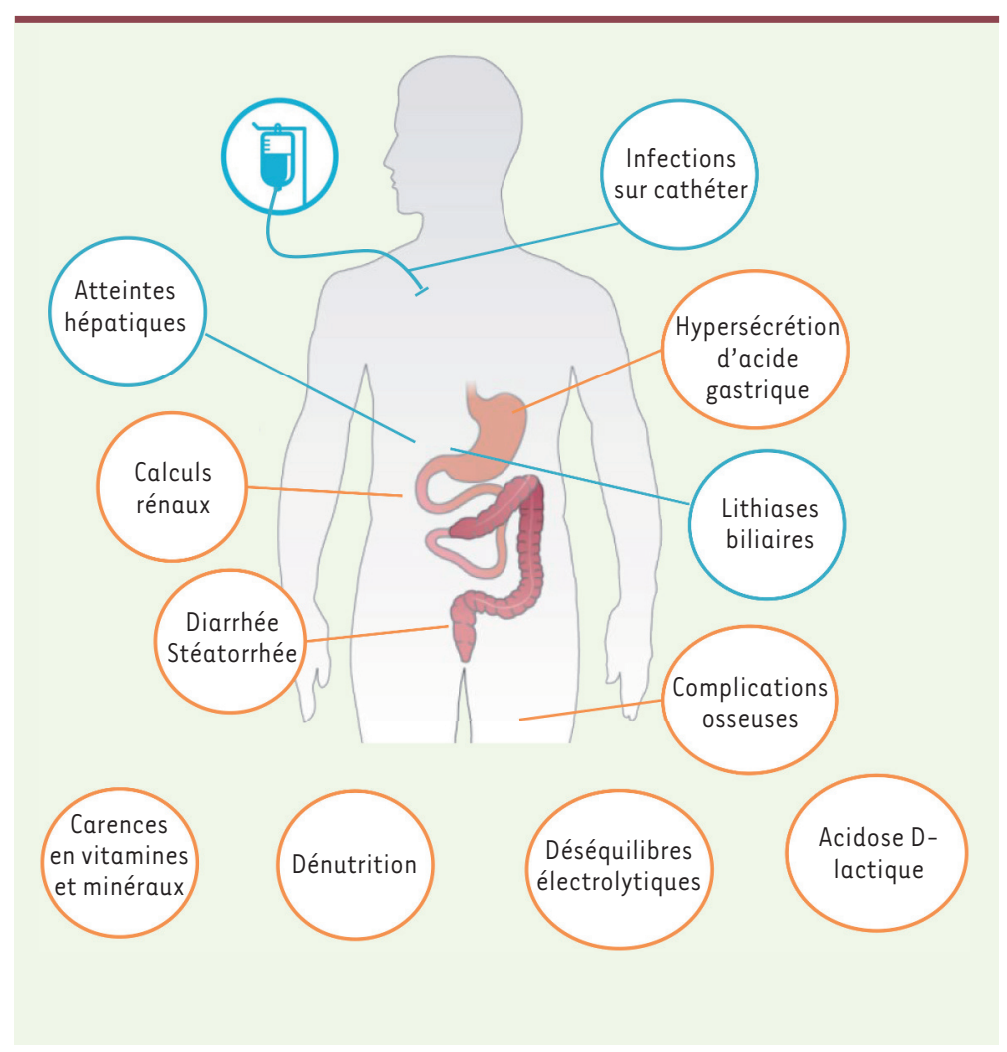

Figure 2. Conséquences pathologiques d'une résection étendue du grêle et effets délétères de la nutrition parentérale à long terme. Une résection étendue du grêle entraîne un certain nombre de conséquences délétères (signalées en orange). La prise en charge par la nutrition parentérale peut également avoir des effets néfastes sur le long terme (en bleu) et diminuer l'espérance de vie des patients atteints de syndrome de grêle court (SGC).

provoque la nutrition parentérale. La diarrhée peut également mettre en jeu le pronostic vital [11].

\section{Malabsorption}

Après une résection intestinale étendue, la malabsorption est d'autant plus importante que la surface d'absorption intestinale résiduelle est réduite. Il en résulte une dénutrition protéinoénergétique (protéines, glucides et lipides) et des carences en vitamines et oligoéléments. Une corrélation entre l'absorption des protéines et des glucides et la longueur de l'intestin grêle résiduelle a été montrée [12], mais la perte de certaines régions intestinales assurant des

rétrocontrôle négatif de la vidange gastrique, notamment par le peptide yy (Pyy) et le GLP-1 (glucagon-like peptide-1). Ces deux entérohormones sont sécrétées par les cellules $L^{3}$ en réponse à l'arrivée d'aliments dans l'iléon et le côlon. Elles permettent de ralentir la vidange gastrique et le transit intestinal [6]. La vidange gastrique est significativement plus lente chez les patients qui ont un côlon résiduel en continuité (types II et III) que chez les patients ayant subi une jéjunostomie (type I). Le transit plus rapide des aliments, incomplètement transformés, voire non transformés, au niveau du grêle alors raccourci, entraîne l'arrivée d'un grand volume de matières souvent hypertoniques dans le côlon. Les pertes d'eau et de sodium sont alors importantes et augmentées par la présence de molécules à fort pouvoir osmotique, comme le lactose. Une hypergastrinémie ${ }^{4}$ apparaît rapidement après la résection. Elle peut contribuer à une diarrhée sécrétoire hydro-électrolytique [7]. Chez les sujets ayant subi une résection iléale étendue (de plus d'un mètre), s'ajoute une entéropathie cholérétique due à une mauvaise ré-absorption des sels biliaires au niveau de l'iléon terminal. Les sels biliaires malabsorbés exercent un effet laxatif sur le côlon et majorent la perte liquidienne $[8,9]$. La perte d'eau et de sel est moins importante pour les types anatomiques II et III car le côlon a une grande capacité d'absorption d'eau et de sodium [10]. Une diarrhée chronique peut persister après la phase aiguë si une malabsorption persiste alors que le transit est ralenti. Ce symptôme est le plus gênant pour le patient, avec un impact négatif sur sa qualité de vie, qui s'avère supérieur à celui que

\footnotetext{
3 Les cellules L sécrètent le glucagon-like peptide-1 (GLP-1), le peptide Yy, l'oxyntomoduline et le glucagon-like peptide-2 (GLP-2). Elles se situent principalement dans l'iléon et le côlon.

${ }^{4}$ Une augmentation anormale de gastrine dans le sang.
} fonctions qui leur sont propres entraîne des carences spécifiques. Ainsi, la perte d'une grande partie de l'iléon (types anatomiques I et II) est responsable d'une malabsorption des lipides et des vitamines liposolubles $(A, D, \varepsilon, K)$, entraînant une stéatorrhée ${ }^{5}$, une carence en vitamine $B 12$ et une rupture du cycle entéro-hépatique $[8,13]$. Dans le type anatomique II, les lipides et les sels biliaires non absorbés sont acheminés vers le côlon, où ils exercent une action irritante sur la muqueuse colique. La perte de la valvule iléo-cæcale a deux conséquences majeures. Sans frein iléal, le transit s'accélère, réduisant l'absorption de l'eau et des nutriments. La résection de la valvule iléo-cæcale entraîne également un reflux des bactéries coliques. Une prolifération microbienne peut en découler, aggravant la diarrhée et la perte de nutriments [14]. La prolifération de certaines bactéries invasives peut alors avoir plusieurs conséquences délétères: la déconjuguaison des acides biliaires, réduisant l'absorption de lipides, la consommation de nutriments comme la vitamine B12, et une action inflammatoire délétère pour l'épithéliim [15]. De plus, un hyperaldostéronisme, secondaire aux pertes hydriques accrues, augmente l'excrétion rénale de magnésium, avec pour conséquence une carence assez fréquente chez les patients [12]. Les carences en vitamines liposolubles peuvent aussi être à l'origine

\footnotetext{
${ }^{5}$ Quantité anormalement élevée de lipides dans les selles.
} 
de coagulopathie ${ }^{6}$, d'ostéoporose, d'atteintes cutanées ou visuelles, etc. L'hypomagnésémie et l'hypokaliémie peuvent être associées à une hypocalcémie, entraînant des troubles neurologiques et, potentiellement, une dysrythmie cardiaque [2].

\section{La phase d'adaptation spontanée}

Après la phase aiguë, des mécanismes d'adaptation se mettent spontanément en place durant les 12 à 24 mois suivant l'installation du SGC, en particulier chez les patients ayant un côlon en continuité de l'intestin grêle. Cette adaptation, qui consiste essentiellement en une hyperphagie et une réponse de l'intestin à la résection, appelée adaptation intestinale, participe à l'amélioration de l'équilibre hydro-électrolytique et énergétique du patient. Elle est stimulée par la présence de nutriments dans le tube digestif et contribue à la diminution, voire à l'arrêt, de la nutrition parentérale.

\section{L'hyperphagie}

Certains patients s'adaptent à la diminution drastique de l'absorption d'énergie en augmentant leurs apports alimentaires [16]. Une hyperphagie, avec des apports supérieurs à 1,5 fois la dépense énergétique basale (DEB), est fréquente et constitue un facteur pronostique favorable chez ces patients [7]. L'accroissement des apports alimentaires permet une augmentation de l'absorption des nutriments, diminuant ainsi la dépendance à la nutrition parentérale. Les mécanismes à l'origine de cette hyperphagie spontanée ne sont cependant pas clairement définis. L'augmentation de la sécrétion de ghréline $e^{7}$ pourrait contribuer à accentuer la sensation de faim (voir plus loin).

\section{L'adaptation intestinale}

$\varepsilon$ n réponse à la résection, l'intestin grêle et éventuellement le côlon résiduels vont présenter deux types de modifications adaptatives: une hyperplasie à l'origine d'une augmentation de la surface d'absorption, et une adaptation fonctionnelle ralentissant le transit et augmentant le temps de contact des nutriments avec la surface d'absorption [17].

\section{Hyperplasie de la muqueuse intestinale}

Une augmentation de la profondeur des cryptes coliques et du nombre de cellules par crypte a été rapportée chez les patients atteints de SGC du type anatomique II [18]. Ces processus, qui augmentent la surface d'absorption, sont favorisés par la présence de nutriments dans l'intestin, par des sécrétions pancréatiques et biliaires ainsi que par des entérohormones [5].

\section{Sécrétion hormonale}

Des concentrations élevées de GLP-1, de GLP-2 et de Pyy ont été observées chez les sujets souffrant d'un SGC de types II et III,

\footnotetext{
6 Défaillance du mécanisme de coagulation du sang.
}

${ }^{7}$ La ghréline est produite et sécrétée dans l'estomac et le duodénum. Cette hormone est connue pour son effet orexigène. Sa concentration sanguine est maximale avant les repas et décroît après l'ingestion des aliments. La ghréline traverse la barrière hémato-encéphalique, puis, par son action sur l'hypothalamus, stimule les mécanismes de la faim. comportant encore un côlon $[19,20]$. Ces entérohormones sont produites par les cellules entéroendocrines de type L situées dans l'iléon et le côlon. Le GLP-2 présente un effet trophique sur l'épithélium intestinal. Son action permet d'augmenter la surface absorbante [21]. Le GLP-1 et le peptide Pyy contribuent au ralentissement de la vidange gastrique, et le GLP-1 ralentit également le transit intestinal $[6,22]$. Des taux élevés de ghréline, une hormone sécrétée essentiellement par des cellules de l'estomac et du duodénum, ont également été rapportés chez les patients atteints du syndrome de grêle court (SGC) [20]. Elle permet d'augmenter la prise alimentaire [20]. Le lien direct entre augmentation de ghréline et hyperphagie reste cependant à démontrer. De même que les mécanismes à l'origine de l'augmentation de la production des différentes entérohormones restent à élucider dans le contexte du SGC.

\section{Le microbiote}

La conservation du côlon permet le maintien d'une flore bactérienne qui pourrait favoriser le sevrage des patients de la nutrition parentérale en augmentant la récupération énergétique de leur alimentation [23, 24]. Chez les patients, l'environnement luminal est profondément modifié par la courte longueur du grêle résiduel (et éventuellement du côlon), la rapidité du transit, la perturbation de la circulation entérohépatique et la grande quantité de nutriments non digérés arrivant dans le côlon. Cet environnement se caractérise par un $\mathrm{pH}$ bas et une teneur élevée en oxygène. La composition du microbiote de ces patients est donc très différente de celle observée chez l'adulte sain, avec une flore dominée principalement par des lactobacilles, qui ont un pouvoir fermentatif élevé permettant de décomposer les glucides en acides gras à chaînes courtes ( $A G C C)$, principale source d'énergie des colonocytes mais également des entérocytes [23]. Ces acides gras à chaînes courtes, ou d'autres métabolites de ce microbiote particulier, peuvent constituer des signaux stimulant d'autres mécanismes d'adaptation, tels que la sécrétion de Pyy $[25,26]$.

Cette phase d'adaptation spontanée, associant une augmentation de la récupération énergétique et hydroélectrolytique, une hyperphagie spontanée et un ralentissement du transit, permet une réduction progressive du niveau de dépendance à la nutrition parentérale avec, pour certains patients, l'acquisition d'une autonomie nutritionnelle par voie orale, même avec un intestin grêle très court. 
La phase stationnaire

Après la période d'adaptation spontanée, les patients entrent dans une phase du syndrome dite stationnaire ou séquellaire. Des complications générales sont alors source de morbidité et de mortalité. II s'agit principalement de risques de dénutrition et de troubles hydro-électrolytiques. Dans le cadre du SGC avec côlon en continuité, les principales complications sont la lithiase oxalique entérique, dont la prévalence peut aller jusqu'à $60 \%$ des patients, et plus rarement l'encéphalopathie D lactique.

\section{Dénutrition et hépatopathie}

Pour contrer le risque de dénutrition et de troubles hydro-électrolytiques, il est nécessaire de réaliser une supplémentation veineuse hydro-électrolytique et/ou calorique. La nutrition parentérale présente des effets délétères à terme, liés aux accès veineux centraux (infections, thromboses veineuses, perte des accès veineux), mais aussi des complications métaboliques (notamment hépatiques, rénales et osseuses). Ces complications sont responsables d'une importante morbidité, ainsi que d'une augmentation du risque de mortalité (avec une survie à 10 ans de $52 \%$ des patients sous nutrition parentérale, toutes causes de SGC confondues). L'hépatopathie, associée à l'insuffisance intestinale, reste une cause importante de mortalité des patients en nutrition parentérale de très longue durée. Elle doit être prévenue dès le stade initial de la constitution du SGC. De nombreux facteurs la favorisent : la présence de segments intestinaux exclus, une colonisation bactérienne, un intestin grêle résiduel de moins de $50 \mathrm{~cm}$, une dénutrition extrême, un déficit en acides aminés (taurine, cystéine, choline), un jeûne prolongé, une nutrition parentérale apportant plus de $1 \mathrm{~g}$ de lipides par jour, une hyperglycémie chronique, un sepsis, ou une lithogenèse ${ }^{8}$ accrue [27]. Ces complications métaboliques sont le plus souvent tardives.

\section{Lithiases rénales d'oxalate de calcium}

La conservation du côlon est un facteur de bon pronostic. Pourtant, dans $25 \%$ des cas, les patients développent des calculs rénaux symptomatiques d'oxalate de calcium [28]. Chez le sujet sain, I'oxalate alimentaire est éliminé sous la forme d'oxalate de calcium et son absorption est inférieure à 10 \% [29]. Chez les patients atteints d'un SGC, les acides gras non absorbés se lient au calcium libérant l'oxalate qui est absorbé au niveau du côlon résiduel puis filtré par les reins. L'oxalate se lie alors au calcium rénal, formant des lithiases pouvant évoluer vers une néphropathie oxalique ou des lithiases obstructives [5].

\section{Prolifération bactérienne et risque d'encéphalopathie D-lactique}

Une prolifération des bactéries dans l'intestin grêle peut s'observer chez les patients ayant conservé leur côlon en l'absence de valvule iléo-cæcale. L'altération de la motilité intestinale participe à cette prolifération bactérienne [15]. Le déséquilibre du microbiote intestinal, qui entraîne une fermentation accrue des résidus alimentaires glucidiques non digérés, produit du D-lactate. Le lactate exogène se

\footnotetext{
${ }^{8}$ Ensemble des processus qui conduisent au développement d'un calcul (lithiase) urinaire ou biliaire.
}

retrouve alors dans le sang et dans les urines, avec un ratio D-lactates (forme exogène) / L-lactates (forme endogène) élevé [30]. Les conséquences de cette acidose D-lactique peuvent aller des ballonnements et des flatulences, désagréables pour les patients, à une encéphalopathie D-lactique, un syndrome neurologique rare caractérisé par un état mental altéré pouvant provoquer de la confusion, des maux de têtes, voire des convulsions et un état comateux [15].

\section{Déminéralisation osseuse}

À long terme, des atteintes osseuses, telles que l'ostéoporose ou l'ostéomalacie, sont des conséquences possibles du SGC. Une étude réalisée sur 167 patients présentant une insuffisance intestinale a en effet révélé une ostéoporose chez plus de la moitié d'entre eux [31]. Cette déminéralisation osseuse peut être expliquée par un déficit de l'apport et une excrétion accrue de calcium, ainsi qu'un défaut de vitamine $D[5,32]$. L'absorption du calcium est fortement altérée par la malabsorption des lipides et par la présence des acides gras dans la lumière intestinale [33].

\section{Prise en charge et suivi des patients}

Pour optimiser la prise en charge des patients, il est nécessaire de tenir compte du type d'anastomose qu'ils ont subi (Figure 1) et de la phase dans laquelle ils se trouvent (Figure 3). Quand cela est possible, une prise en charge rapide et adaptée doit donc être organisée dès l'intervention chirurgicale.

\section{Prise en charge nutritionnelle}

Outre le traitement ciblant la cause ayant conduit à l'intervention, il est important de prendre en charge les conséquences digestives de la résection intestinale. En phase aiguë, la prise en charge de l'hypersécrétion gastrique acide est nécessaire, via la prise d'inhibiteurs de la pompe à protons (IPP) [7]. Ce traitement pourra être arrêté après un an pour les patients de types anatomiques II ou III, mais il peut être nécessaire de le poursuivre à vie pour les patients ayant subi une jéjunostomie [7]. Les pertes de fluides et d'électrolytes étant également importantes, les solutions orales de réhydratation, en plus du support nutritionnel, sont alors recommandées afin de pouvoir compenser rapidement la déshydratation [7]. Dès que le patient est en condition stable, en phase stationnaire, une nutrition parentérale est mise en place afin de couvrir ses besoins énergétiques et de compenser les pertes. Des supplémentations en calcium, magnésium, zinc et vitamine $D$ sont administrées si nécessaire $[34,2]$. En 


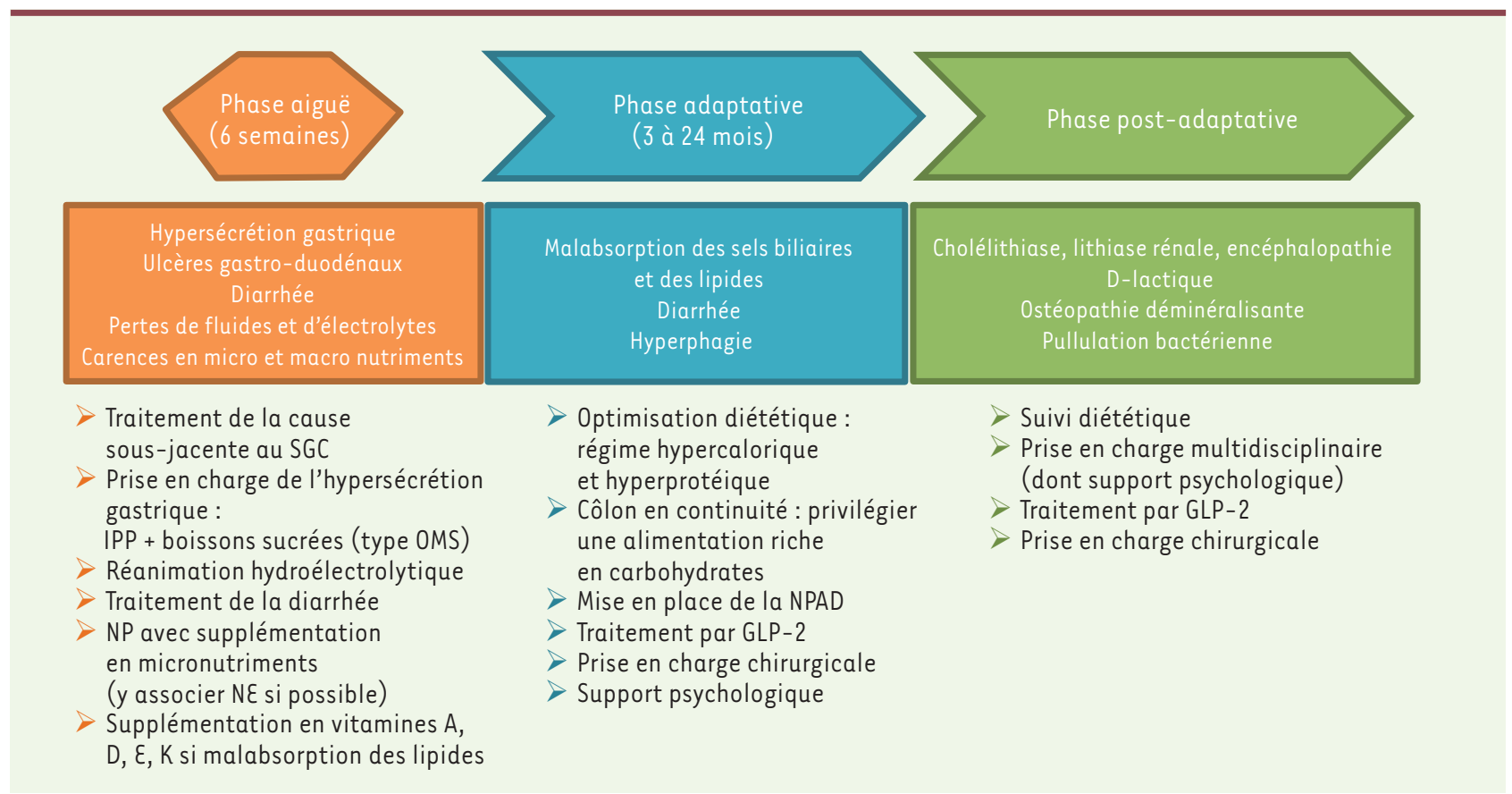

Figure 3. Recommandations pour la prise en charge des patients atteints d'un syndrome de grêle court (SGC) selon la phase de la maladie et les symptômes. La prise en charge nutritionnelle des sujets atteints dépend à la fois du type de résection et de la phase de la maladie. NP : nutrition parentérale; NE : nutrition entérale; NPAD : nutrition parentérale à domicile; OMS : Organisation mondiale de la santé ; IPP : inhibiteurs de la pompe à protons.

cas de malabsorption des lipides, une supplémentation en vitamines liposolubles ( $A, D, \varepsilon, K$ ) est également administrée [7]. La durée de la nutrition parentérale dépend de la longueur du grêle restant, de la localisation de la résection, et de la présence ou non d'un côlon en continuité [5]. Certains facteurs prédictifs de la dépendance à la nutrition parentérale ont été rapportés: la longueur de grêle restant et sa fonctionnalité, évaluée par la concentration plasmatique précoce de citrulline (un acide aminé spécifiquement synthétisé par les entérocytes fonctionnels), la présence du côlon en continuité, et le niveau d'hyperphagie compensatrice [35]. Dans le cas où les ingesta du patient sont faibles, une nutrition entérale, par sonde gastrique ou intestinale, combinée ou non à la nutrition parentérale, permet d'éviter l'atrophie de la muqueuse intestinale, de préserver le microbiote et, peut-être, de stimuler l'hyperphagie spontanée [5].

La prise en charge diététique des patients doit être évaluée dès que possible afin de compenser la malabsorption. L'hyperphagie est encouragée avec une alimentation si possible solide hypercalorique, riche en protéines (20\% de l'apport calorique) [36]. Les lipides étant moins bien tolérés chez les patients avec un côlon en continuité, une alimentation pauvre en lipides et riche en carbohydrates est généralement conseillée à ces patients. En revanche, l'alimentation hyperlipidique est recommandée chez les patients ne possédant pas de côlon en continuité [2] $]^{9}$. Plusieurs traitements, en particulier pour ralentir le transit et prévenir la diarrhée, sont préconisés, notamment la prise

${ }^{9}$ Contrairement aux glucides qui sont absorbés massivement dans la partie proximale de l'intestin grêle, l'absorption lipidique suit une progression linéaire jusqu'à l'iléon. de lopéramide ou de phosphate de codéine (des antidiarrhéiques) [5, 7]. Des antibiotiques peuvent aussi être nécessaires pour certains patients, notamment en cas de prolifération bactérienne [7]. Néanmoins, il convient de rester très prudent sur l'antibiothérapie: celle-ci peut en effet modifier de manière importante le microbiote, déstabiliser la flore colique et aggraver la diarrhée.

\section{Complications dues à la nutrition parentérale}

Bien que la nutrition parentérale soit le traitement de référence pour les patients présentant un SGC, cette dernière peut avoir un impact important sur leur qualité de vie et être à l'origine de complications. L'infection liée au cathéter veineux central (ILCVC) nécessaire à la nutrition parentérale représente l'une de ses principales complications. Les infections et thromboses sur cathéter augmentent significativement la mortalité des patients sous nutrition parentérale [37]. Les multiples manipulations de la ligne veineuse, du cathéter et du pansement, sont autant de facteurs de risque de survenue d'une ILCVC [38]. Des solutions à base de taurolidine (un antibiotique) peuvent cependant être instillées en verrou dans la lumière du cathéter afin de limiter la contamination bactérienne [39], ce qui réduit significativement le risque infectieux à domicile [40]. 
En cas de résection iléale étendue, la formation de calculs biliaires affecte un patient sur deux [28]. L'incidence de cette affection est liée à la durée de la nutrition parentérale [41]. Plusieurs facteurs ont été identifiés pour expliquer la formation de ces calculs, dont la stase biliaire [2]. Le déficit en acides biliaires augmente le risque de formation de dépôts de cholestérol, associé au bicarbonate de calcium et à la bilirubine [28]. Ces lithiases peuvent être asymptomatiques ou se révéler de façon brutale par une cholestase ou une pancréatite, justifiant une cholécystectomie ${ }^{10}$ prophylactique chez les patients ayant une nutrition parentérale de long cours [2]. Les atteintes hépatiques des patients imputables à ce type de nutrition peuvent être à l'origine de différentes manifestations cliniques. Leur physiopathologie est multifactorielle [42] : inflammation intrahépatique associée à une surcharge hépatique en graisses, aboutissant à une stéatohépatite non alcoolique (ou NASH) ; apport d'acides gras polyinsaturés à longue chaîne $\omega-6$ qui sont des précurseurs de médiateurs de l'inflammation ; et altération du cycle entérohépatique. Les infections récurrentes, ainsi que les translocations bactériennes ou d'endotoxines provenant de segments exclus, peuvent également contribuer à ces atteintes hépatiques [40]. La stéatose peut évoluer en fibrose, nécessitant dans certains cas une transplantation hépatique [43]. À noter que la survie des patients présentant une insuffisance intestinale chronique sous nutrition parentérale de longue durée est de de $88 \%$ à un an, $74 \%$ à 3 ans et $64 \%$ à 5 ans [44].

\section{La prise en charge intégrée dans un parcours de soins}

Si la nutrition parentérale à domicile est prolongée au-delà de douze semaines, le patient (adulte) nécessite un suivi médical par un centre agréé ou expert appartenant à un établissement de soins [40]. L'équipe, constituée de médecins, de pharmaciens, d'infirmiers et de diététiciens, et dirigée par un médecin ayant l'expertise dans la prise en charge de l'insuffisance intestinale sévère et en nutrition parentérale, doit être formée à la prise en charge de la nutrition parentérale, notamment à l'éducation thérapeutique. Le centre assure la formation du patient et/ou des personnes qui réaliseront la pratique de la technique à domicile.

\section{Prise en charge chirurgicale}

Les patients avec entérostomie doivent être évalués en vue d'un éventuel rétablissement de la continuité digestive afin d'optimiser la longueur du circuit digestif en maintenant, si possible, le côlon [7]. Ce rétablissement permet d'améliorer le pronostic et la prise en charge du patient. L'absence de stomie proximale diminue les risques de déshydratation et de troubles hydro-électrolytiques, mais surtout, la remise en continuité du côlon diminue la dépendance du patient à la nutrition parentérale et améliore ainsi la survie à longterme. Ce rétablissement de la continuité est souvent proposé entre trois à six mois après la dernière chirurgie de résection, afin de faciliter le geste de rétablissement et diminuer le risque de morbidité postopératoire. Ce rétablissement de continuité digestive est suf-

\footnotetext{
10 Ablation de la vésicule biliaire.
}

fisant pour obtenir un sevrage de la nutrition parentérale chez la plupart des patients présentant un SGC de type anatomique II (ayant conservé la majorité du côlon dans le circuit digestif, mais sans valvule iléocæcale) avec un minimum de $65 \mathrm{~cm}$ d'intestin grêle restant, ou présentant un SGC de type anatomique III (ayant conservé $100 \%$ du côlon dans le circuit digestif, y compris la valvule iléo-cæcale), avec un minimum de $75 \mathrm{~cm}$ d'intestin grêle restant [7].

Des méthodes de chirurgie reconstructrice, comme celle de l'anse jéjunale antipéristaltique, peuvent être envisagées pour optimiser l'absorption par l'intestin grêle restant. Cette chirurgie consiste à inverser un segment, d'environ $10 \mathrm{~cm}$, de l'intestin grêle, afin d'augmenter le temps de transit des nutriments [45]. Elle doit être considérée comme une option dans la prise en charge du SGC sans iléon, en particulier dans le cas d'un risque important d'une insuffisance intestinale permanente [7].

Pour les patients chez lesquels le pronostic à court terme est engagé, en raison de l'échec de la nutrition parentérale (patients présentant une hépatopathie sévère, ou en cas de perte des axes veineux centraux), la possibilité de la greffe intestinale peut être envisagée. Cette intervention reste néanmoins extrêmement rare et peu pratiquée en raison d'un risque élevé de mortalité [7].

\section{Prise en charge pharmacologique}

\section{Les traitements disponibles}

Afin de réduire les risques liés à l'administration de la nutrition parentérale et d'améliorer la qualité de vie des patients, des traitements visant à améliorer l'absorption intestinale ont été développés. Les hormones ayant une activité trophique sur l'épithélium intestinal ont été particulièrement étudiées et font aujourd'hui partie de l'arsenal thérapeutique du SGC.

Le premier traitement mis en place a été un traitement par l'hormone de croissance, en association avec une nutrition particulière riche en glutamine. Ce traitement, qui a démontré son efficacité, est actuellement utilisé aux États-Unis. L'administration de l'hormone de croissance permet en effet de réduire significativement la nutrition parentérale chez les patients, la combinaison avec la glutamine permettant de maintenir l'effet du traitement [46].

Le deuxième traitement à base d'hormone repose sur I'utilisation d'un analogue du GLP-2. Le GLP-2 stimule la croissance intestinale (augmentation de la taille des villosités et de la profondeur des cryptes), renforce la fonction barrière intestinale et ralentit la vitesse du 
transit des aliments [21]. Sa demi-vie est cependant réduite (environ sept minutes) et sa clairance rénale importante [21], le rendant difficile d'utilisation en clinique. Des analogues ayant une demi-vie plus longue ont donc été développés ces dernières années.

Le téduglutide (commercialisé en Europe sous le nom REVESTIVદ®) est le premier (et pour le moment le seul) analogue du GLP-2 ayant obtenu une autorisation de mise sur le marché (AMM) pour le traitement du SGC. II s'agit d'un peptide qui comporte dans sa séquence, par rapport au GLP-2, une substitution de l'alanine en position 2 par une glycine, ce qui le rend résistant à la dégradation par la dipeptidyl peptidase-4. II possède ainsi une demi-vie plus longue que celle du GLP-2 (environ deux heures) [47]. Dans le premier essai thérapeutique sur le téduglutide, une réduction d'au moins $20 \%$ du volume hebdomadaire de la nutrition parentérale a été observée chez $93 \%$ des patients traités pendant 30 mois et quatre patients ont été sevrés de la nutrition parentérale [48]. Après sa mise sur le marché, le téduglutide a fait l'objet de quelques études en vie réelle. Une patiente a présenté des adénomes du grêle (un dans le duodénum et un dans le jéjunum) après 41 mois de traitement [49], suggérant que des effets indésirables pourraient être observés après une durée prolongée de traitement et qu'il est important d'obtenir des facteurs prédictifs de réponse ou non au traitement.

\section{Les traitements en cours d'évaluation}

Compte tenu de la contrainte liée à la posologie du téduglutide (nécessité d'une injection quotidienne), d'autres analogues du GLP-2 ayant des demi-vies plus longues ont été développés. Le glépaglutide se différencie du GLP-2 par la substitution de neuf acides aminés dans sa séquence et par une extrémité $C$-terminale constituée de six résidus lysine. Ces modifications permettent d'augmenter sa demi-vie à près de 50 heures, réduisant ainsi le nombre d'injections hebdomadaires nécessaires. Une étude clinique multicentrique, dont la phase III est en cours (elle a commencé en octobre 2018) (ClinicalTrials.gov Identifier: NCT03905707), présente des résultats prometteurs, notamment en ce qui concerne l'amélioration de l'absorption intestinale, l'hydratation des patients et la fonction rénale. Cependant, le traitement semble avoir des effets indésirables hépatiques, avec notamment une activation des macrophages hépatiques qui reflète une inflammation cellulaire [50]. L'apraglutide est en cours de développement. Ce peptide analogue du GLP-2 se caractérise par la substitution de quatre acides aminés $\left(\mathrm{Ala}^{2}>\mathrm{Gly}, \mathrm{Met}^{10}>\mathrm{Ahx}, \mathrm{Asn}{ }^{11}>\mathrm{D}\right.$ Phe, et $A s n^{16}>$ Leu). Sa clairance, très faible, a été évaluée in vivo chez le rat [51]. Sa posologie consiste en une injection par semaine, ce qui présente un avantage important par rapport aux autres analogues. Des essais cliniques de phases I/II ont démontré son efficacité. Comparé au glépaglutide, l'apraglutide présente une meilleure sélectivité vis-à-vis du récepteur du GLP-2 (comparable à celle du téduglutide), et semble avoir un effet trophique plus important. Un essai de phase III est en cours (ClinicalTrials.gov Identifier : NCT04627025) [51].

Comme le GLP-2, le GLP-1 est une entérohormone qui dérive du proglucagon, à l'origine du glucagon dans le pancréas, et du GLP-l et du GLP-2 dans l'intestin. L'exénatide est un agoniste du récepteur du GLP1. Sa séquence présente $53 \%$ d'identité avec celle du GLP-1 humain.
Il est utilisé depuis 2005 pour le traitement du diabète de type 2. Une étude pilote réalisée avec cinq patients a permis de montrer une réduction des mouvements contractiles intestinaux chez les patients traités, et d'obtenir un sevrage de la nutrition parentérale pour trois d'entre eux [52]. Ce traitement pourrait permettre une amélioration des capacités d'absorption de l'intestin chez les patients atteints d'un SGC. L'administration de co-agonistes (GLP-1 et GLP-2) est également en cours d'étude. Ces différents traitements sont prometteurs, mais ils nécessitent, du fait de leur développement récent, des études de tolérance et d'efficacité à long terme.

\section{Les autres pistes thérapeutiques}

Des stratégies thérapeutiques développées pour des maladies différentes pourraient être appliquée dans le cadre du syndrome de grêle court (SGC). C'est le cas de la transplantation de microbiote fécal (TMF), qui consiste en l'introduction de la flore bactérienne d'un donneur sain dans le tube digestif d'un patient receveur. Elle est utilisée pour traiter des infections récurrentes à Clostridium difficile, réfractaires aux antibiothérapies, mais également dans le cadre de $\mathrm{MICl}$, de troubles fonctionnels intestinaux et de maladies métaboliques dans lesquelles une dysbiose (perturbation du microbiote) serait impliquée [53]. Une modification profonde de la composition du microbiote est observée lors de la phase d'adaptation du SGC. Le microbiote du patient chez lequel cette modification a lieu semble contribuer à une meilleure récupération énergétique [54]. On pourrait ainsi imaginer la transplantation de ce microbiote déjà « adapté » à un patient en phase précoce du SGC.

Le taux de réussite des transplantations intestinales reste faible [7]. Aussi, la greffe de cellules souches intestinales, pour remplacer les portions d'intestin non fonctionnelles, est maintenant envisagée [55]. Élaborer en laboratoire un intestin fonctionnel prêt à être greffé aux patients présentant un SGC permettrait d'éviter les contraintes liées au besoin de donneur ainsi que le recours aux immunosuppresseurs.

\section{Conclusion}

Le syndrome de grêle court est une maladie rare mais complexe, résultant de différentes causes et présentant différentes formes anatomiques. Sa prise en charge est donc délicate, et la compréhension des mécanismes adaptatifs spontanés survenant chez les patients après une résection intestinale étendue est fondamentale pour tenter de réduire l'impact de la maladie et de son traitement sur leur qualité de vie. Cette prise en charge, 
qui doit être multidisciplinaire, repose sur une surveillance étroite, une supplémentation nutritionnelle adaptée, et un suivi thérapeutique qui devrait déjà avoir été planifié avant la résection chirurgicale de l'intestin. Elle permet aujourd'hui une espérance de vie élevée pour les patients atteints du syndrome de grêle court. $\diamond$

\section{SUMMARY}

Short bowel syndrome: From intestinal insufficiency to intestinal adaptation

The short bowel syndrome results from an extensive intestinal resection. When intestinal function is below the minimum necessary for the absorption of macronutrients, water and electrolytes, short small bowel syndrome is responsible for chronic intestinal failure. The management is then parenteral nutrition. The evolution of the short bowel syndrome is schematically divided into three successive periods: (a) Immediate postoperative period lasting 3 to 6 weeks; (b) adaptive period lasting about 2 years and (c) stabilization period. However, the development of hyperphagia, spontaneous intestinal adaptation allowing an increase in the absorption surface area and in secretion of enterohormones and a modification of the microbiota occur spontaneously, improving intestinal absorption and decreasing dependence on parenteral nutrition. This review summarizes the main positive and negative pathophysiological consequences of extensive intestinal resection and the nutritional and drug management of short bowel syndrome in adults. $\diamond$

\section{LIENS D'INTÉRÊT}

Les auteures déclarent n'avoir aucun lien d'intérêt concernant les données publiées dans cet article.

\section{RÉFÉRENCES}

1. Tappenden KA. Pathophysiology of short bowel syndrome. J Parenter Enter Nutr 2014 ; 38 : S14-22.

2. Pironi L, Corcos 0 , Forbes $A$, et al. Intestinal failure in adults: recommendations from the ESPEN expert groups. Clin Nutr Edinb Scotl $2018 ; 37: 1798-809$.

3. Boland $\varepsilon$, Thompson J, Rochling F, et al. A 25 -year experience with postresection short-bowel syndrome secondary to radiation therapy. Am J Surg $2010 ; 200: 690-3$.

4. Jeppesen PB. Spectrum of short bowel syndrome in adults: intestinal insufficiency to intestinal failure. J Parenter Enter Nutr 2014 ; 38 : S8-13.

5. Massironi S, Cavalcoli F, Rausa $\varepsilon$, et al. Understanding short bowel syndrome: current status and future perspectives. Dig Liver Dis 2020 ; 52 : 253-61.

6. Nightingale JM, Kamm MA, Sijp JR van der, et al. Gastrointestinal hormones in short bowel syndrome. Peptide YY may be the colonic brake to gastric emptying. Gut 1996 ; 39 : 267-72.

7. Billiauws L, Maggiori L, Joly F, et al. Medical and surgical management of short bowel syndrome.J Visc Surg 2018; 155 : 283-91.

8. Hofmann AF, Poley JR. Role of bile acid malabsorption in pathogenesis of diarrhea and steatorrhea in patients with ileal resection. I. Response to cholestyramine or replacement of dietary long chain triglyceride by medium chain triglyceride. Gastroenterology $1972 ; 62$ : 918-34.

9. Camilleri M, Sellin JH, Barrett KE. Pathophysiology, evaluation, and management of chronic watery diarrhea. Gastroenterology 2017 ; 152 : 515-32.e2.

10. Debongnie JC, Phillips SF. Capacity of the human colon to absorb fluid. Gastroenterology 1978 ; $74: 698-703$.

11. Winkler MF, Hagan $\varepsilon$, Wetle T, et al. An Exploration of quality of life and the experience of living with home parenteral nutrition. J Parenter Enter Nutr 2010 ; 34 : 395-407.

12. Nightingale JMD. Management of patients with a short bowel. World J Gastroenterol $2001 ; 7: 741-51$

13. Jeejeebhoy KN. Short bowel syndrome: a nutritional and medical approach. Can Med Assoc J 2002 ; $166: 1297-302$.

14. Gracey M. The contaminated small bowel syndrome: pathogenesis, diagnosis, and treatment. Am J Clin Nutr $1979 ; 32$ : 234-43.

15. DiBaise JK, Young RJ, Vanderhoof JA. Enteric microbial flora, bacterial overgrowth, and shortbowel syndrome. Clin Gastroenterol Hepatol $2006 ; 4: 11-20$.

16. Crenn P. Net digestive absorption and adaptive hyperphagia in adult short bowel patients. Gut $2004 ; 53: 1279-86$.
17. Porus RL. Epithelial hyperplasia following massive small bowel resection in man. Gastroenterology $1965 ; 48: 753-7$.

18. Joly F, Mayeur C, Messing B, et al. Morphological adaptation with preserved proliferation/transporter content in the colon of patients with short bowel syndrome. Am J Physiol Gastrointest Liver Physiol 2009 ; 297 : G116-23.

19. Jeppesen P, Hartmann B, Thulesen J, et al. Elevated plasma glucagon-like peptide 1 and 2 concentrations in ileum resected short bowel patients with a preserved colon. Gut $2000 ; 47: 370-6$.

20. Gillard L, Billiauws L, Stan-luga B, et al. Enhanced ghrelin levels and hypothalamic orexigenic agrp and npy neuropeptide expression in models of jejuno-colonic short bowel syndrome. Sci Rep 2016; 6 : 28345.

21. Brubaker PL. Glucagon-like peptide- 2 and the regulation of intestinal growth and function. Compr Physiol 2018; $8: 26$.

22. Martin GR, Wallace $L E$, Hartmann $B$, et al. Nutrient-stimulated GLP-2 release and crypt cell proliferation in experimental short bowel syndrome. Am J Physiol Gastrointest Liver Physiol 2005 ; 288 : G431-8.

23. Briet $F$, Flourié $B$, Achour $L$, et al. Bacterial adaptation in patients with short bowel and colon in continuity. Gastroenterology $1995 ; 109$ : 1446-53.

24. Nordgaard I, Hansen BS, Mortensen PB. Colon as a digestive organ in patients with short bowel. Lancet 1994 ; 343 : 373-6.

25. Brooks L, Viardot A, Tsakmaki A, et al. Fermentable carbohydrate stimulates FFAR2-dependent colonic Pyy cell expansion to increase satiety. Mol Metab $2017 ; 6: 48-60$.

26. Wichmann A, Allahyar A, Greiner TU, et al. Microbial modulation of energy availability in the colon regulates intestinal transit. Cell Host Microbe 2013; 14 : 582-90.

27. Morgan J, Dibb M, Lal S. Intestinal failure-associated liver disease in adult patients. Curr Opin Clin Nutr Metab Care 2019 ; 22 : 383-8.

28. Nightingale JM, Lennard-Jones JE, Gertner DJ, et al. Colonic preservation reduces need for parenteral therapy, increases incidence of renal stones, but does not change high prevalence of gall stones in patients with a short bowel. Gut $1992 ; 33$ : 1493-7.

29. Smith LH, Fromm H, Hofmann AF. Acquired hyperoxaluria, nephrolithiasis, and intestinal disease. Description of a syndrome. N Engl J Med 1972 ; 286 : 1371-5.

30. Mayeur C, Gratadoux JJ, Bridonneau C, et al. Faecal D/L lactate ratio is a metabolic signature of microbiota imbalance in patients with short bowel syndrome. PLoS One $2013 ; 8$ : e54335.

31. Nygaard L, Skallerup A, Olesen SS, et al. Osteoporosis in patients with intestinal insufficiency and intestinal failure: prevalence and clinical risk factors. Clin Nutr 2018 ; 37 : 1654-60.

32. Bikle DD. Vitamin $D$ Insufficiency/deficiency in gastrointestinal disorders.J Bone Miner Res 2007 ; 22 : V50-4.

33. Hylander $\varepsilon$, Ladefoged K, Madsen S. Calcium balance and bone mineral content following small-intestinal resection. Scand J Gastroenterol 1981 ; 16 : 167-76.

34. Ladefoged K, Nicolaidou P, Jarnum S. Calcium, phosphorus, magnesium, zinc, and nitrogen balance in patients with severe short bowel syndrome. Am J Clin Nutr $1980 ; 33: 2137-44$.

35. Amiot A, Messing B, Corcos 0 , et al. Determinants of home parenteral nutrition dependence and survival of 268 patients with non-malignant short bowel syndrome. Clin Nutr 2013 ; 32 : 368-74.

36. Matarese $L \varepsilon$. Nutrition and fluid optimization for patients with short bowel syndrome. J Parenter Enter Nutr 2013 ; 37 : 161-70.

37. Messing B, Crenn P, Beau P, et al. Long-term survival and parenteral nutrition dependence in adult patients with the short bowel syndrome. Gastroenterology 1999 ; 117 : 1043-50.

38. Dibb M, Lal S. Home parenteral nutrition: vascular access and related complications. Nutr Clin Pract 2017 ; 32 : 769-76.

39. Daoud DC, Wanten G, Joly F. Antimicrobial locks in patients receiving home parenteral nutrition. Nutrients $2020 ; 12: 439$.

40. Pironi L, Boeykens K, Bozzetti F, et al. ESPEN guideline on home parenteral nutrition. Clin Nutr Edinb Scotl 2020 ; 39 : 1645-66.

41. Manji N, Bistrian BR, Mascioli EA, et al. Gallstone disease in patients with severe short bowel syndrome dependent on parenteral nutrition.J Parenter Enteral Nutr 1989; 13 : 461-4.

42. Pironi L, Goulet 0 , Buchman A, et al. Outcome on home parenteral nutrition for benign intestinal failure: a review of the literature and benchmarking with the European prospective survey of ESPEN. Clin Nutr 2012;31:831-45.

43. Cavicchi M, Beau P, Crenn P, et al. Prevalence of liver disease and contributing factors in patients receiving home parenteral nutrition for permanent intestinal failure. Ann Intern Med 2000 ; 132 : 525-32. 


\section{RÉFÉRENCES}

44. Joly F, Baxter J, Staun M, et al. Five-year survival and causes of death in patients on home parenteral nutrition for severe chronic and benign intestinal failure. Clin Nutr 2018 ; 37 : 1415-22

45. Beyer-Berjot L, Joly F, Maggiori L, et al. Segmental reversal of the small bowel can end permanent parenteral nutrition dependency: an experience of 38 adults with short bowel syndrome. Ann Surg $2012 ; 256: 739-44$.

46. Byrne TA, Wilmore DW, lyer $K$, et al. Growth hormone, glutamine, and an optimal diet reduces parenteral nutrition in patients with short bowel syndrome. Ann Surg $2005 ; 242: 655-61$.

47. Jeppesen PB, Sanguinetti $\varepsilon L$, Buchman A, et al. Teduglutide (ALX-0600), a dipeptidyl peptidase IV resistant glucagon-like peptide 2 analogue, improves intestinal function in short bowel syndrome patients. Gut $2005 ; 54: 1224-31$

48. Seidner DL, Fujioka K, Boullata JI, et al. Reduction of parenteral nutrition and hydration support and safety with long-term teduglutide treatment in patients with short bowel syndromeassociated intestinal failure: STEPS-3 study. Nutr Clin Pract 2018 ; 33 : 520-7.

49. Pevny S, Pape UF, Elezkurtaj S, et al. De novo development of distal jejunal and duodenal adenomas after 41 months of teduglutide treatment in a patient with short-bowel syndrome: a case report. J Parenter Enteral Nutr $2021 ; 45: 652-6$.

50. Hvistendahl MK, Naimi RM, Enevoldsen LH, et al. Effect of glepaglutide, a long-acting glucagonlike peptide-2 analog, on gastrointestinal transit time and motility in patients with short bowel syndrome: findings from a randomized trial. J Parenter Enteral Nutr 2020 ; 44 : $1535--44$.
51. Hargrove DM, Alagarsamy S, Croston G, et al. Pharmacological characterization of apraglutide, a novel long-acting peptidic glucagon-like peptide-2 agonist, for the treatment of short bowel syndrome. J Pharmacol Exp Ther $2020 ; 373: 193-203$.

52. Kunkel D, Basseri B, Low K, et al. Efficacy of the glucagon-like peptide-l agonist exenatide in the treatment of short bowel syndrome. Neurogastroenterol Motil 2011; $23: 7$.

53. Danne C, Rolhion N, Sokol H. Recipient factors in faecal microbiota transplantation: one stool does not fit all. Nat Rev Gastroenterol Hepatol 2021 ; doi: 10.1038/s41575-021-00441-5.

54. Gillard L, Mayeur C, Robert V, et al. Microbiota Is involved in post-resection adaptation in humans with short bowel syndrome. Front Physiol 2017 ; 8 : 224.

55. Sugimoto $S$, Kobayashi $\varepsilon$, Fujii $M$, et al. An organoid-based organrepurposing approach to treat short bowel syndrome. Nature $2021 ; 592$ : 99-104.

\section{www.myobase.org}

Catalogue en ligne disponible gratuitement sur Internet publié par l'AFM-Téléthon.

Retrouvez facilement toutes les références bibliographiques sur les maladies neuromusculaires, les situations de handicap qu'elles génèrent et leurs aspects psychologiques.

\section{Myobase donne un accès libre à $75 \%$ du \\ UN OUTIL ERGONOMIQUE, UNE INTERFACE BILINGUE} fonds documentaire collecté depuis 1990 , représentant plus de 40000 références spécifiques du domaine des maladies neuromusculaires.

$>$ articles de la littérature biomédicale et psycho-sociale

\section{$>$ livres, thèses}

$>$ guides d'associations et rapports institutionnels d'agences internationales

\section{$>$ brèves en français,} synthèses des articles médico-scientifiques internationaux les plus pertinents

\section{$>$ publications}

AFM-Téléthon destinées aux professionnels de santé ou aux personnes atteintes de maladie neuromusculaire et à leur entourage
- Laissez-vous guider par les tutoriels

- Lancez une recherche et affinez votre sélection grâce aux filtres

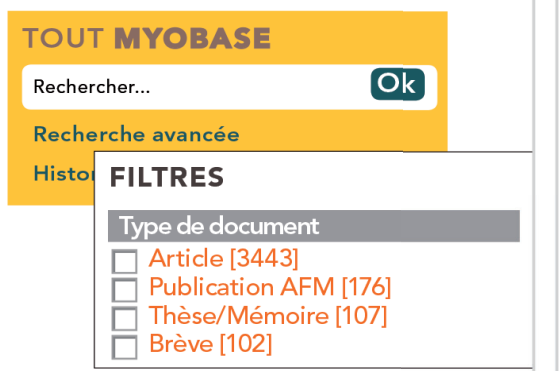

- PUBLICATIONS AFM-Téléthon

- BRÈVES

- DOCUMENTS DE SYNTHÈsE

INSTITUT DES BIOTHÉRAPIES PUBLICATIONS

- Partagez les résultats de votre recherche
UN ACCÈS facile et simple

Rechercher avec des opérateurs :

- guillemets pour une expression "maladie de pompe"

- + pour signifier ET, et retrouver tous les documents contenant les deux mots "fauteuil +électrique"

- - pour signifier NON et enlever le mot de la recherche:

"autonomie -établissement"
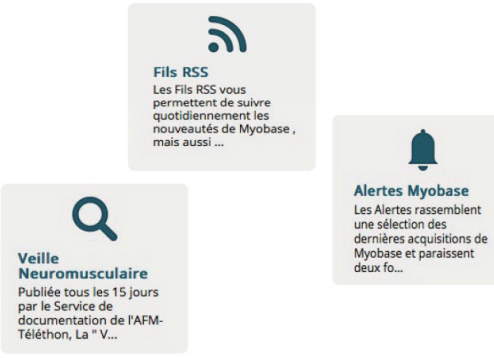

- Cliquez sur l'onglet thématique qui vous convient (haut de la page d'accueil)

- Créez vos alertes personnalisées en ouvrant un compte personnel

- Téléchargez la Veille Neuromusculaire

- Abonnez-vous aux flux RSS 